\title{
Assessment of Acute Pancreatitis Severity and Prognosis with CT-Measured Body Composition
}

\author{
Ying Zhou ${ }^{1,2, *}$ \\ Ningbo $\mathrm{Hao}^{2, *}$ \\ Zhongping Duan ${ }^{3}$ \\ Ming Kong ${ }^{3}$ \\ Manman $\mathrm{Xu}^{3}$ \\ Dan Zhang ${ }^{2}$ \\ Xiangxiang $\mathrm{Xu}^{2}$ \\ Qingwen Yuan ${ }^{1,2}$ \\ Changzheng $\mathrm{Li} \mathbb{D}^{2}$
}

'Postgraduate Training Base of Jinzhou Medical University, Jinzhou, People's

Republic of China; ${ }^{2}$ Department of Gastroenterology and Hepatology, The PLA Rocket Force Characteristic Medical Center, Beijing, People's Republic of China; ${ }^{3}$ Beijing Municipal Key Laboratory of Liver Failure and Artificial Liver Treatment Research, You'an Hospital Affiliated to Capital Medical University, Beijing, People's Republic of China

*These authors contributed equally to this work

\begin{abstract}
Objective: The aim of this study was to investigate the possible association of muscle and adipose parameters with the severity and prognosis of patients hospitalized with acute pancreatitis (AP).

Methods: A total of 392 hospitalized patients and 309 controls were enrolled in the study analysis from April 1, 2016, to February 1, 2021. The computed tomography scans of each population were evaluated for muscle and adipose parameters. The effects of parameters on developing moderately severe acute pancreatitis (MSAP) or severe acute pancreatitis (SAP) were evaluated using univariate and multivariate logistic regression analyses. Associations with disease recurrence and death were analyzed through Cox regression analysis.
\end{abstract}

Results: The AP patients had higher levels of visceral adipose tissue (144.25 vs $97.81 \mathrm{~cm}^{2}, \mathrm{p}<$ 0.001 ) and subcutaneous adipose tissue ( $135 \mathrm{vs} 120 \mathrm{~cm}^{2}, \mathrm{p}<0.001$ ) but lower levels of adipose tissue attenuation (visceral and subcutaneous) and skeletal muscle attenuation (SMA) than the controls ( $\mathrm{p}<0.05$, respectively). Visceral adipose tissue (VAT) and SMA differed significantly with $\mathrm{p}$-values of 0.014 and 0.003 in the different severity groups of AP. In multivariate analysis, VAT and SMA were associated with MSAP or SAP, with odds ratios of 1.003 and 0.973 , respectively ( $95 \%$ CI $1.000-1.006, p=0.041 ; 95 \%$ CI $0.953-0.993, p=0.010)$. Cox regression analysis showed that low SMA was strongly associated with an increased mortality in MSAP and SAP patients (HR $10.500,95 \%$ CI 1.344-82.025, $\mathrm{p}=0.025$ ). Regression analysis also showed an association of VAT loss of more than $17 \%$ with reduced 1 -year recurrence of acute pancreatitis (HR $0.427,95 \% \mathrm{CI}$ $0.189-0.967, \mathrm{p}=0.041$ ).

Conclusion: VAT and SMA were influential factors for the severity and prognosis of patients with AP. Patients should proper diet and exercise after discharge to reduce VAT and strengthen muscle function to improve prognosis.

Keywords: skeletal muscle attenuation, visceral adipose tissue, subcutaneous adipose tissue, recurrent acute pancreatitis

\section{Plain Language Summary}

It is well known that the incidence, recurrence and mortality of acute pancreatitis are increasing year by year. Computed tomography (CT) imaging is a method of accurately quantifying abdominal adipose and skeletal muscle parameters and is deemed the gold standard for body composition assessment. We collected CT images from 392 patients with acute pancreatitis and 309 controls. We found that patients with acute pancreatitis had higher visceral adipose tissue (VAT) and lower skeletal muscle attenuation (SMA) than the control groups. High VAT is a risk factor for moderately severe acute pancreatitis (MSAP) or severe acute pancreatitis (SAP). On the contrary, high SMA was a protective factor for MSAP or SAP. We also found low SMA was strongly associated with an increased mortality rate in MSAP and SAP patients. VAT loss of more than $17 \%$ were associated with reduced recurrence of acute pancreatitis at 1 year. It is our opinion that patients should receive education after discharge from the hospital. Proper diet and exercise
Correspondence: Changzheng $\mathrm{Li}$ Department of Gastroenterology and Hepatology, The PLA Rocket Force Characteristic Medical Center, 16 Xinjiekouwai Street, Beijing, I00088, People's Republic of China

Email licz007@aliyun.com 
can reduce VAT and increase the muscle function of patients, which is beneficial for improving the quality of life and prognosis of patients.

\section{Introduction}

Acute pancreatitis (AP), a major aseptic inflammatory state of the pancreas, is frequently caused by gallstones, alcohol consumption, hypertriglyceridemia or the presence of other rare etiologies. The worldwide incidence of acute pancreatitis is 34 cases per 100,000 persons, and the number of hospitalizations has gradually increased in recent years. ${ }^{1,2}$ A majority of those with hospitalizations due to AP make a full recovery, but it is noteworthy that approximately $17-29 \%$ of patients have recurrent AP (RAP) after complete or near recovery, and 3\% to $14 \%$ of these patients develop chronic pancreatitis (CP). ${ }^{3}$ Some recent reports indicated that the mortality rate was approximately $2 \%$ in patients with moderately severe acute pancreatitis (MSAP) and significantly increased (36-50\%) in patients with severe acute pancreatitis (SAP). ${ }^{4,5}$ The poor prognosis of AP poses a huge burden on the medical system. It has been reported that recurrent AP can reduce the physical and mental quality of life despite the absence of CP. ${ }^{6}$

Computed tomography (CT) imaging is a method of accurately quantifying abdominal adipose and skeletal muscle parameters and is deemed the gold standard for body composition assessment. ${ }^{7,8}$ Abdominal CT was performed in almost all AP patients. Abdominal CT on admission and clinical scoring systems were similar in assessing the severity of AP. ${ }^{9}$ A recent systematic review has shown a strong association between increased visceral adipose tissue and the severity of AP, although it is not an independent risk factor for AP. ${ }^{10}$ In contrast, low skeletal muscle attenuation (SMA) is a risk factor for SAP rather than visceral adipose tissue (VAT). ${ }^{11}$ For prognostic analysis, recent studies have suggested that a decrease in skeletal muscle density $\geq 10 \%$ in 1 month was significantly associated with increased inhospital mortality in patients with necrotizing pancreatitis. ${ }^{12}$ Low SMA was significantly associated with worse overall survival. There are few studies on the risk factors for recurrent AP in the literature, especially in Asian countries.

The primary aim of our current study was to explore whether body composition parameters were influential factors in the occurrence of MSAP or SAP. Notably, we analyzed whether body composition loss was associated with patients with recurrent AP. A secondary aim was to assess their impact on short-term mortality in patients with MSAP and SAP.

\section{Methods}

\section{Study Population}

In this retrospective study, we collected 392 hospitalized patients with acute pancreatitis and 303 controls from the PLA Rocket Force Characteristic Medical Center in China between April 1, 2016, and February 1, 2021. The diagnosis of AP included a combination of symptoms, physical examination, important laboratory and imaging tests, with two of the following three criteria being met: 1) upper abdominal pain of an acute and persistent nature often radiating through to the back, 2) serum amylase or lipase levels 3 times higher than the upper limit of normal, and 3) abdominal CT scans consistent with the appearance of acute pancreatitis. ${ }^{13}$ The severity of AP was divided into three types, namely, mild, moderately severe, and severe, according to the different clinical manifestations and prognoses in the Atlanta Classification 2012 revision. ${ }^{13}$ Mild AP patients presented with no organ failure and local/systemic complications. The fatality rate of MAP is extremely low. Moderately severe AP patients had transient organ failure that recovered within 48 hours and/or local or systemic complications. Severe AP was defined as persistent single or multiple organ failure ( $>48$ hours duration). Individuals with pancreatitis were excluded in the study if they have (1) malignancy; (2) hepatitis, liver cirrhosis and other serious liver diseases; (3) chronic pancreatitis. A total of 303 controls had only simple gastrointestinal polyps, mild gastroesophageal reflux disease, chronic gastritis, or dyspepsia. The exclusion criteria were (1) acute and chronic pancreatitis; (2) malignant diseases; (3) endocrine and metabolic syndrome, such as thyroid dysfunction and diabetes mellitus; (4) various of serious diseases, such as liver failure, respiratory and circulatory failure, renal failure; (5) autoimmune diseases; (6) various of chronic diseases, such as cardio-cerebra vascular diseases, Chronic hepatitis and liver cirrhosis. For patients who were seen in the hospital for recurrent AP more than once during the survey period, the first recurrence was used for further analysis. Mortality was determined from clinical case records. Time of death in days between the date of CT and the date of death was recorded.

\section{CT Image Analysis}

In this research, abdominal CT scans were completed in all patients and controls. All CT images were obtained from the PACS system and measured in dicom format. CT images were selected at the middle level of the third lumbar vertebrae and 
measured using sliceOmatic software (version 5.0; Tomovision, Magog, Canada) to quantify body tissues. ${ }^{14}$ Different tissues are divided according to different density thresholds, such as -29 to +150 Hounsfield units (HU) representing skeletal muscle tissue (SMT), -150 to -50 HU representing VAT and -190 to -30 representing subcutaneous adipose tissue (SAT). A trained operator then manually sketches out each tissue. Figure 1 shows skeletal muscle in red, visceral adipose tissue in yellow and subcutaneous adipose tissue in green. The area and mean attenuation (HU) of different tissues were calculated automatically. The crosssectional skeletal muscle area was normalized to height $\left(\mathrm{cm}^{2} / \mathrm{m}^{2}\right)$ to evaluate the skeletal muscle index (SMI). Skeletal muscle attenuation (SMA) was determined by the mean Hounsfield unit (HU) value of all pixels included in SMT and correlated with the triglyceride content of skeletal muscle. $^{15}$

\section{Statistical Analysis}

We used SPSS statistics software 26 (version 26.0; IBM Corp., Armonk, NY, UAS) to analyze our data and make diagrams. A P-value less than 0.05 was statistically significant, and a P-value less than 0.1 was marginally significant. Categorical variables are expressed as numbers (percentages) and were compared by the chi-square test. Continuous data are shown as the median (interquartile range [IQR]), and the Kolmogorov-Smirnov test was used to examine the normality of the data distribution. We used Mann-Whitney $U$-tests or Kruskal-Wallis $H$-tests for comparisons between groups in the case of nonnormally distributed data. Post hoc analysis was executed using Bonferroni adjustment when statistically significant differences were observed among the three groups. Univariate and multivariate logistic regression analyses were applied to evaluate the risk factors for MSAP or
SAP. P-values lower than 0.1 in univariate analysis and considered statistically significant were included in multivariate analysis. For the prognosis analyses, all body parameters were classified into high and low subgroups using the median value of different tissues as the cutoff. Stepwise Cox regression analysis was applied to identify body composition indexes significantly associated with 3-month survival in patients with MSAP and SAP. Similarly, we used Cox regression analysis to observe whether the rate of loss in body composition was a risk factor for 1-year recurrence.

\section{Results}

\section{Baseline Features and Body Composition Analysis in Controls and Patients with Acute Pancreatitis}

The sample comprised 392 patients $(61.5 \%$ male; median age 54 years [IQR 40-71]) and 309 controls (62.1\% male; median age 49 years [IQR 36-61]). The cause of AP was biliary in $189(48.21 \%)$ patients, hyperlipidemia in 74 (18.88\%) patients, alcoholic in $21(5.36 \%)$ patients, and other in 108 (27.55\%) patients. The number of acute pancreatitis patients with hypertension and diabetes was 144 and 86, respectively. 341 had a first attack and 51 had a previous history of acute pancreatitis. With respect to the characteristics of body composition. BMI was significantly higher in AP patients than in controls (24.98 [IQR 22.49-28.06] vs 23.36 [IQR 21.4625.75]; $\mathrm{p}<0.001)$. AP patients had significantly higher VAT (144.25 [IQR 95.10-198.88] vs 97.81 [IQR 39.54167.00]; $\mathrm{p}<0.001)$ and SAT (135.00 [IQR 101.53-185.20] vs 120.00 [IQR 87.95-161.50]; $\mathrm{p}<0.001)$ but significantly lower VATA, SATA and SMA when compared to controls ( $\mathrm{p}$ $<0.05$ for each; Table 1). The study showed that patients with higher adipose tissue and lower attenuation (muscle and adipose tissue) were more likely to develop AP.
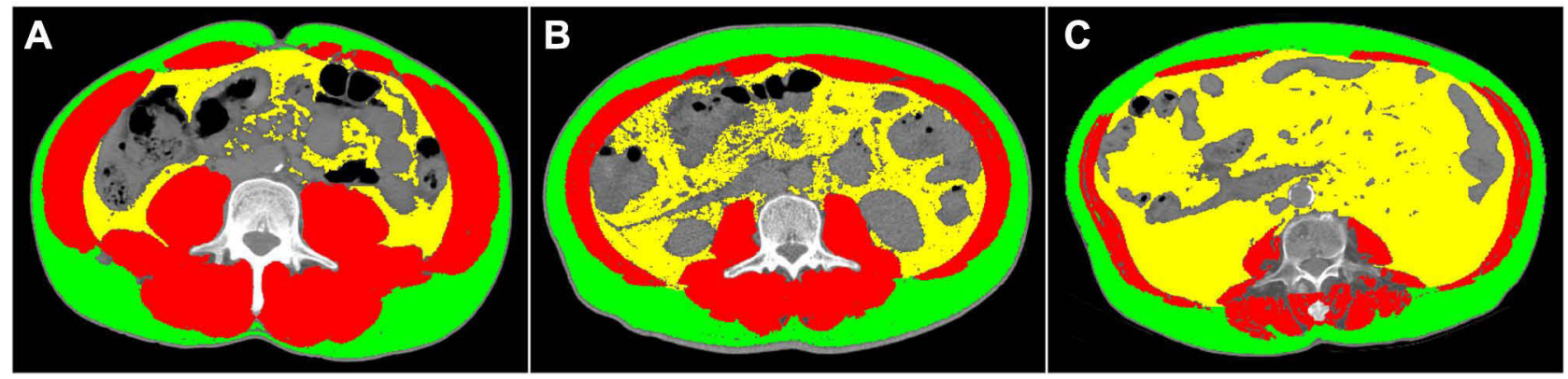

Figure I Computed tomographic scans showing areas of body composition in AP patients with different severities. (A-C) Show mild, moderately severe, and severe AP, respectively. Skeletal muscle is represented in red, visceral adipose tissue is represented in yellow, and subcutaneous adipose tissue is represented in green. At a similar BMI, as the severity of AP increased, VAT also increased gradually $\left(54.01 \mathrm{~cm}^{2}, 135.8 \mathrm{~cm}^{2}\right.$ and $\left.372.4 \mathrm{~cm}^{2}\right)$. In contrast, SMA showed a decreasing trend $(52.4 \mathrm{I} \mathrm{HU,} 37.08 \mathrm{HU}$ and I8.99 HU). 
Table I Baseline Features of Control and Patients with Acute Pancreatitis

\begin{tabular}{|c|c|c|c|}
\hline Parameters & $\operatorname{AP}(n=392)$ & Control $(n=309)$ & $P$-value \\
\hline Male sex, $\mathrm{n}(\%)$ & $24 I(6 I .5)$ & $192(62.1)$ & 0.859 \\
\hline Age(years) & $54(40-7 I)$ & $49(36-6 I)$ & $<0.001$ \\
\hline $\operatorname{BMI}\left(\mathrm{kg} / \mathrm{m}^{2}\right)^{\mathrm{a}}$ & $24.98(22.49-28.06)$ & $23.36(21.46-25.75)$ & $<0.001$ \\
\hline $\operatorname{VAT}\left(\mathrm{cm}^{2}\right)^{\mathrm{a}}$ & |44.25(95.10-198.88) & $97.81(39.54-167.00)$ & $<0.001$ \\
\hline $\operatorname{VATA}(H U)^{a}$ & $-94.24(-98.82 \sim-87.22)$ & $-92.15(-97.13 \sim-83.33)$ & 0.002 \\
\hline $\operatorname{SAT}\left(\mathrm{cm}^{2}\right)^{\mathrm{a}}$ & $135.00(101.53-185.20)$ & $120.00(87.95-161.50)$ & $<0.001$ \\
\hline $\operatorname{SATA}(H U)^{a}$ & $-99.22(-103.1 \sim-93.76)$ & $-98.01(-101.45 \sim-91.92)$ & 0.003 \\
\hline $\operatorname{SMT}\left(\mathrm{cm}^{2}\right)^{\mathrm{a}}$ & $123.00(98.59-\mid 60.88)$ & $128.30(103.65-156.37)$ & 0.491 \\
\hline $\mathrm{SMA}(\mathrm{HU})^{\mathrm{a}}$ & $38.73(30.94-44.57)$ & $45.13(38.62-50.07)$ & $<0.001$ \\
\hline L3-SMI $\left(\mathrm{cm}^{2} / \mathrm{m}^{2}\right)^{\mathrm{a}}$ & $43.66(37.62-55.20)$ & $44.50(38.59-51.99)$ & 0.842 \\
\hline Smoking, n(\%) & $93(23.7)$ & $70(22.7)$ & 0.739 \\
\hline Drinking, $\mathrm{n}(\%)$ & $98(25)$ & $62(20.1)$ & 0.122 \\
\hline \multicolumn{4}{|l|}{ Etiology, n(\%) } \\
\hline Biliary & $189(48.2 \mid)$ & & \\
\hline Hyperlipidemia & $74(18.88)$ & & \\
\hline Alcoholic & $2 I(5.36)$ & & \\
\hline Other & $108(27.55)$ & & \\
\hline Hypertension, n(\%) & $133(33.93)$ & & \\
\hline Diabetes, $n(\%)$ & $86(21.94)$ & & \\
\hline Previous AP history & $5 I(13.01)$ & & \\
\hline
\end{tabular}

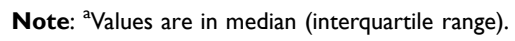

\section{Comparison of Different Body Variables According to Severity of Acute Pancreatitis and Prediction of Risk Factors for MSAP or SAP}

Table 2 shows that we enrolled 178, 161 and 53 patients with mild, moderately severe and severe AP, respectively. The median age increased as the AP severity increased, but it was not significantly different among the three groups. The sex distribution was also not significantly different. SMA (39.90 [IQR 98.67-154.15], 38.37 [IQR 30.28-44.41] and 32.25 [IQR 26.59-41.36]; $\mathrm{p}=0.003)$ and VAT (138.8 [IQR 92.08-183.43], 150.2 [IQR 90.22202.25] and 166.4 [IQR 112.65-224.65]; $\mathrm{p}=0.014)$ were significantly different among the three groups. By post hoc analysis, SMA and VAT were significantly higher in the severe group than in the mild group $(\mathrm{p}=0.002, \mathrm{p}=0.013)$. An example of a CT photograph taken at mid-L3 of AP patients with a similar BMI is shown in Figure 1. Interestingly, as the severity of AP increased, VAT also

Table 2 Comparison of Body Composition Variables According to Severity of Acute Pancreatitis

\begin{tabular}{|c|c|c|c|c|}
\hline \multirow[t]{2}{*}{ Variables } & \multicolumn{3}{|c|}{ Severity of Acute Pancreatitis } & \multirow[t]{2}{*}{$P$-value } \\
\hline & Mild(n=I78) & Moderately Severe $(n=|6|)$ & Severe $(n=53)$ & \\
\hline Male sex, $\mathrm{n}(\%)$ & $104(58.4)$ & $107(66.5)$ & $30(56.6)$ & 0.232 \\
\hline Age $(\text { years })^{a}$ & $53(4 \mid-68)$ & $54(36-71)$ & $63(41.5-79)$ & 0.122 \\
\hline $\mathrm{BMI}(\mathrm{kg} / \mathrm{m} 2)^{\mathrm{a}}$ & $24.67(22.49-27.35)$ & $25.33(22.24-28.38)$ & $26.04(22.67-29.05)$ & 0.249 \\
\hline $\operatorname{VAT}\left(\mathrm{cm}^{2}\right)^{\mathrm{a}}$ & $138.8(92.08-183.43)$ & $150.2(90.22-202.25)$ & $166.4(|| 2.65-224.65)^{b}$ & 0.014 \\
\hline $\operatorname{VATA}(\mathrm{HU})^{\mathrm{a}}$ & $-95.25(-99.23 \sim-87.81)$ & $-92.96(-98.24 \sim-86.02)$ & $-95.16(-99.39 \sim-88.34)$ & 0.203 \\
\hline $\operatorname{SAT}\left(\mathrm{cm}^{2}\right)^{\mathrm{a}}$ & $134.2(102.63-180.78)$ & $|3| .5(97.02-185.60)$ & $147.5(107.15-197.4)$ & 0.354 \\
\hline $\operatorname{SATA}(H U)^{a}$ & $-99.19(-103.00 \sim-95.25)$ & $-98.99(-103.1 \sim-92.82)$ & $-99.63(-104.85 \sim-91.54)$ & 0.677 \\
\hline $\operatorname{SMT}\left(\mathrm{cm}^{2}\right)^{\mathrm{a}}$ & $119.5(98.67-154.15)$ & $128.3(101.8-167.3)$ & $111.5(94.73-151.85)$ & 0.169 \\
\hline $\operatorname{SMA}(H U)^{\mathrm{a}}$ & $39.90(32.78-45.7 I)^{\mathrm{b}}$ & $38.37(30.28-44.4 I)$ & $32.25(26.59-41.36)^{\mathrm{b}}$ & 0.003 \\
\hline L3-SMI $\left(\mathrm{cm}^{2} / \mathrm{m}^{2}\right)^{\mathrm{a}}$ & $42.20(37.70-51.34)$ & $45.64(38.24-56.66)$ & $40.73(36.58-54.23)$ & 0.106 \\
\hline
\end{tabular}

Notes: ${ }^{a}$ Values are in median (interquartile range). ${ }^{b}$ Severe versus mild were statistical significance by post hoc analysis using Bonferroni adjustment. 
increased gradually $\left(54.01 \mathrm{~cm}^{2}, 135.8 \mathrm{~cm}^{2}\right.$ and $372.4 \mathrm{~cm}^{2}$ ). In contrast, SMA showed a decreasing trend (52.41 HU, 37.08 HU and 18.99 HU).

The results from the univariate and multivariate regression analyses for MSAP or SAP are shown in Table 3. The majority of variables did not significantly correlate with risk factors for MSAP or SAP. However, SMA (OR 0.973, 95\% CI 0.953-0.993, $\mathrm{p}=0.01$ ) and VAT (OR 1.003, 95\% CI 1.000-1.006, $\mathrm{p}=0.041)$ were associated with developing MSAP or SAP. Specifically, our data suggested that a oneunit increase in visceral adipose tissue was associated with a $0.3 \%$ increase in risk factors for MSAP or MAP. A oneunit increase in skeletal muscle attenuation was associated with a $2.7 \%$ decrease in risk factors for MSAP or MAP.

\section{Associations of Body Parameters with MSAP and SAP with Respect to 3-Month Mortality}

Among our population, there were no deaths in patients with mild AP. It has been reported in the literature that the mortality of MAP patients is also extremely low, ${ }^{13}$ so we only observed the risk factors for short-term mortality for MSAP (dead/alive, 2/161, 1.2\%) or SAP (dead/alive, 9/53, $17 \%$ ) patients. For the prognosis analyses, all body parameters were entered as above and below subgroups using the median value of different tissues as the cutoff. We also included repeat acute pancreatitis and previous medical history (Hypertension and Diabetes). 181 had a first attack and 33 had repeat pancreatitis. For 3-month survival, patients with high skeletal muscle attenuation had a mortality rate of $0.9 \%$ (dead/alive, $1 / 107$ ) compared to 9.3\% (dead/alive, 10/107) among patients with low skeletal muscle attenuation. According to the stepwise Cox regression model, the 3-month survival risk was significantly associated with low skeletal muscle attenuation (hazard ratio [HR]: $10.500,95 \%$ CI: 1.344-82.025, $\mathrm{p}=0.025)$. Figure 2 displays the 3 -month survival curves for MSAP and SAP patients according to high or low skeletal muscle attenuation.

\section{Associations of Body Composition Parameters with AP with Respect to I-Year Recurrence}

In this population, 79 patients underwent CT review, including 33 patients with recurrence and 46 patients without recurrence. Table 4 shows the baseline CT and followup CT of the patients at 1 year. In the nonrecurrent group, the median VAT decreased from 144.20 (IQR 101.35209.80) $\mathrm{cm}^{2}$ to 103 (IQR 69.68-162.38) $\mathrm{cm}^{2}$ ( $\mathrm{p}=0.005$ ), which was statistically significant. However, the median VAT was not significantly different between the first AP and recurrent AP groups (156.6 [IQR 119.70-198.85] vs 152 [IQR 100.3-186.7], $\mathrm{p}=0.442$ ). Similarly, the decrease in SAT from 129.60 (IQR 95.06-192.78) $\mathrm{cm}^{2}$ to 114.55 (IQR 72.07-170.40) $\mathrm{cm}^{2} \quad(\mathrm{p}=0.055)$ was marginally significant.

Table 3 Univariate and Multivariate Logistic Regression Analysis for Risk Factors for MSAP or SAP

\begin{tabular}{|c|c|c|c|c|}
\hline \multirow[t]{2}{*}{ Parameters } & \multicolumn{2}{|c|}{ Univariate } & \multicolumn{2}{|c|}{ Multivariate } \\
\hline & OR $(95 \% \mathrm{Cl})$ & $P$-value & OR $(95 \% \mathrm{Cl})$ & $P$-value \\
\hline Male sex & $1.266(0.842-1.905)$ & 0.258 & & \\
\hline Age & $1.006(0.995-1.016)$ & 0.286 & & \\
\hline BMI & $1.028(0.979-1.080)$ & 0.272 & & \\
\hline VAT & $1.004(1.001-1.006)$ & 0.009 & $1.003(1.000-1.006)$ & 0.041 \\
\hline VATA & $1.018(0.996-1.041)$ & 0.115 & & \\
\hline SAT & $1.001(0.999-1.004)$ & 0.313 & & \\
\hline SATA & $1.012(0.989-1.036)$ & 0.293 & & \\
\hline SMT & $1.002(0.997-1.007)$ & 0.501 & & \\
\hline SMA & $0.969(0.949-0.989)$ & 0.002 & $0.973(0.953-0.993)$ & 0.010 \\
\hline L3-SMI & $1.008(0.990-1.025)$ & 0.396 & & \\
\hline Smoking & $0.740(0.46 \mathrm{I}-\mathrm{I} .188)$ & 0.213 & & \\
\hline Drinking & $0.697(0.437-I . I I I)$ & 0.129 & & \\
\hline Hypertension & $0.855(0.56 \mathrm{I}-\mathrm{I} .304)$ & 0.467 & & \\
\hline Diabetes & $0.736(0.452-1.197)$ & 0.217 & & \\
\hline Previous AP history & $0.617(0.334-1.138)$ & 0.122 & & \\
\hline
\end{tabular}




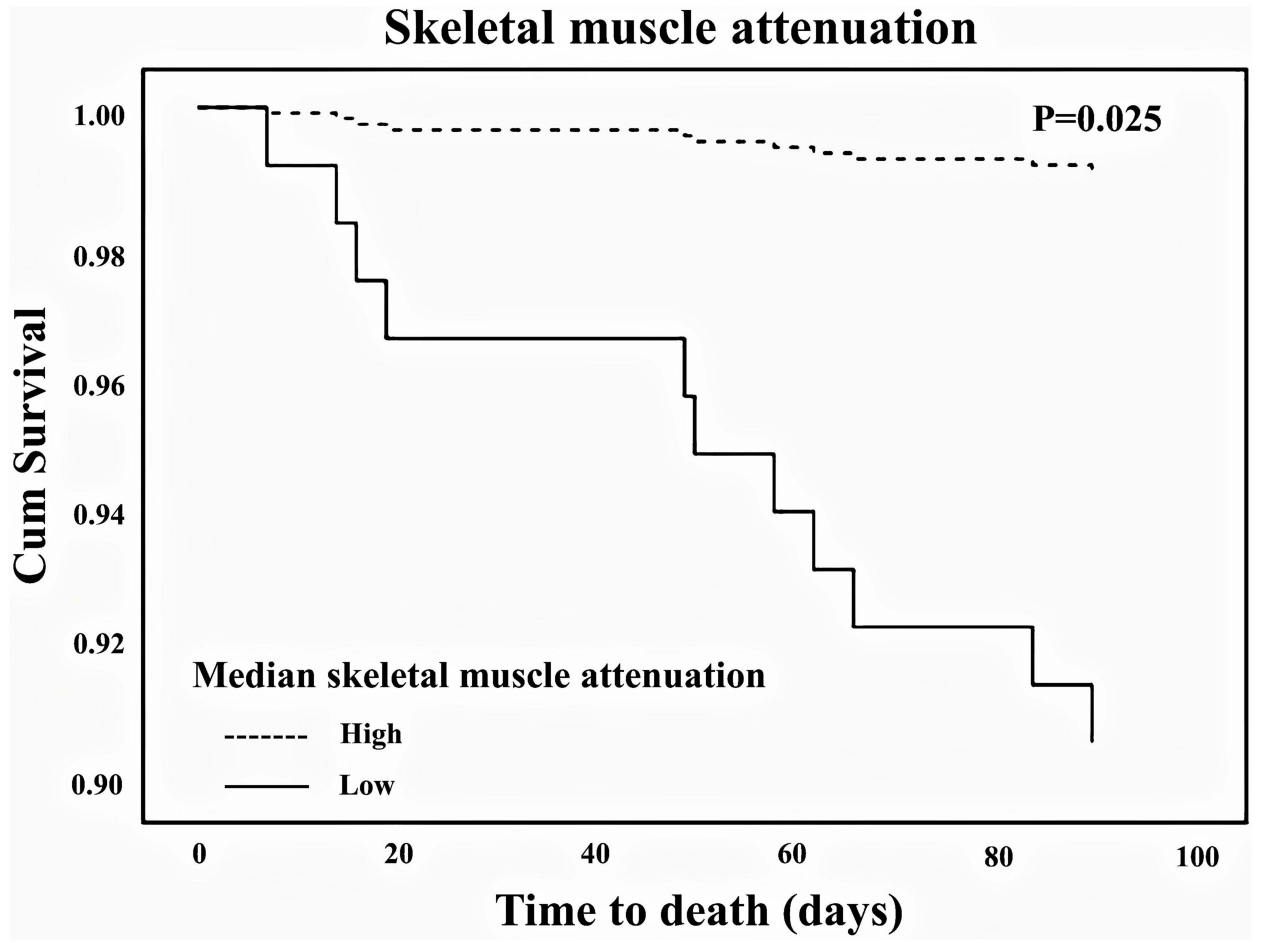

Figure 2 Stepwise cox model 3 month survival curves of high and low skeletal muscle attenuation. The mortality rate for patients with high skeletal muscle attenuation was $0.9 \%$ (dead/alive, $1 / 107$ ) and for those with low skeletal muscle attenuation was $9.3 \%$ (dead/alive, 10/107). Cox regression analysis of body composition showed that only low skeletal muscle attenuation was a risk factor for 3-month mortality (hazard ratio [HR]: 10.500, 95\% Cl: 1.344-82.025, $\mathrm{p}=0.025$ ).

The rate of body composition loss (\%) was equal to the ratio between the difference value of two CT scans (a baseline CT and a follow-up CT) and the first body composition value. The median percentage of VAT decrease was $17 \%$, and the recurrence rate was $23 \%$ (9/39) among patients with more than $17 \%$ visceral adipose tissue loss compared to $60 \%(24 / 40)$ among patients with less than $17 \%$ visceral adipose tissue loss. Hazard ratios were determined for the development of RAP as ascertained by Cox regression analysis. The results showed that more than $17 \%$ visceral adipose tissue loss (hazard ratio [HR] 0.427; 95\% CI 0.189$0.967, \mathrm{P}=0.041$ ) was a protective factor for 1 -year recurrence (Table 5).

\section{Discussion}

Body composition parameter analysis is an emerging method and is easy to obtain from computed tomography (CT) images. CT has become increasingly important in the diagnosis, treatment, and follow-up of patients with AP. Our research showed that CT-assessed VAT and SMA were significantly associated with the development of MSAP or SAP. SMA was associated with the mortality rate in patients with MSAP and SAP. Notably, visceral adipose tissue loss of $\geq 17 \%$ within 1 year was a protective factor for recurrent AP. Body composition might be the key to predicting clinical outcomes.

Few studies on AP have included control groups. This study showed that patients with AP had lower adipose

Table 4 Body Composition Features of Recurrent and No-Recurrent with AP by Different States in I Year

\begin{tabular}{|c|c|c|c|c|c|c|}
\hline & \multicolumn{2}{|l|}{ No-Recurrent( $n=46)$} & \multirow[t]{2}{*}{ P-value } & \multicolumn{2}{|l|}{ Recurrent(n=33) } & \multirow[t]{2}{*}{ P-value } \\
\hline & Initial(AP) & Initial(Normal) & & Initial(AP) & Initial(RAP) & \\
\hline VAT & |44.20(I0|.35-209.80) & $103.00(69.68-162.38)$ & 0.005 & $156.60(119.70-198.85)$ & $152.00(100.30-186.70)$ & 0.442 \\
\hline SAT & $129.60(95.06-192.78)$ & II $4.55((72.07-170.40)$ & 0.055 & I52.00(I I0.70-182.25) & |48.30(97.47-|85.65 & 0.577 \\
\hline SMT & $118.90(97.15-145.80)$ & $106.05(93.38-|4| .98)$ & 0.410 & $126.80(95.49-162.30)$ & $129.70(91.02-157.10)$ & 0.858 \\
\hline SMA & $37.42(28.69-43.30)$ & $35.74(29.92-43.90)$ & 0.821 & $37.67(31.56-44.31)$ & $40.28(30.84-44.69)$ & 0.677 \\
\hline L3-SMI & $41.20(37.43-50.15)$ & $39.4 I(36.33-48.65)$ & 0.290 & $42.86(37.25-57.07)$ & $43.16(35.46-56.03)$ & 0.893 \\
\hline
\end{tabular}


Table 5 Association Between Rate of Body Composition Loss with in I Year Recurrent, According to Univariate Cox Regression Analysis

\begin{tabular}{|l|l|l|l|}
\hline Parameters & Hazard Ratio & 95\% Confidence Interval & P-value \\
\hline Male sex & 0.110 & $0.5 I I-2.413$ & $0.79 I$ \\
Age & 0.995 & $0.977-1.013$ & 0.572 \\
VAT rate of loss (>median) & 0.427 & $0.189-0.967$ & $0.04 I$ \\
SAT rate of loss (>median) & 0.604 & $0.295-1.238$ & 0.168 \\
SMT rate of loss (>median) & 0.887 & $0.429-1.832$ & 0.745 \\
SMA rate of loss (>median) & 0.874 & $0.436-1.754$ & 0.705 \\
L3-SMI rate of loss (>median) & 0.887 & $0.429-I .832$ & 0.745 \\
\hline
\end{tabular}

tissue attenuation than controls. Low adipose tissue attenuation corresponds to larger adipocytes and a higher lipid content. ${ }^{16}$ This was consistent with the conclusion that both subcutaneous (135 vs $120 \mathrm{~cm}^{2}$ ) and visceral (144.25 vs $97.81 \mathrm{~cm}^{2}$ ) adipose tissue were higher in AP patients than in the control group. The AP patients also had lower SMA than the control group (38.73 vs 45.13 $\mathrm{HU})$. Lower CT attenuation of muscle is usually due to increased fatty infiltration of muscle, known as "myosteatosis". 17 A low level of SMA, indicating myosteatosis, was shown to be a major factor affecting muscle function. These results suggested that high adipose tissue and low attenuation (muscle and adipose tissue) may be risk factors for developing AP.

In this study, SMA was an influential factor in the occurrence of MSAP or SAP. A one-unit increase in skeletal muscle attenuation was associated with a 3.1\% decrease in risk factors for MSAP or MAP. We found only one study showing that low mean muscle attenuation was a risk factor for SAP, but it did not explain its impact on the severity of AP. ${ }^{11}$ Our possible explanations are as follows. Recent studies have suggested that high levels of skeletal muscle attenuation were positively correlated with high levels of leptin. ${ }^{18}$ Leptin is secreted by adipose tissue and also by skeletal muscle. ${ }^{19}$ The main function of leptin is to act on the hypothalamus to suppress appetite and limit lipid deposition in peripheral tissues, including skeletal muscle. ${ }^{20,21}$ High levels of leptin would prevent lipid accumulation in muscle, thus maintaining high skeletal muscle attenuation. Leptin can also play an antiinflammatory role. $^{22}$ Patients with low SMA have decreased skeletal muscle function. At the same time, decreased leptin produced by skeletal muscle results in reduced anti-inflammatory effects. It has been reported that exogenous leptin supplementation in animal experiments can decrease the level of inflammation and reduce the expression of proinflammatory factors and NO, thereby reducing the severity of pancreatitis. ${ }^{22}$ We also explored risk factors for mortality in MSAP and SAP patients. The mortality rate in this group of patients was 5.1\% (11/214), which is similar to the reported rate of approximately $5.4 \%{ }^{23}$ It has been reported that CT-assessed low SMA was strongly associated with mortality from cirrhosis, esophageal cancer and extremity sarcomas. ${ }^{24-26}$ It has also been reported that low skeletal muscle attenuation is associated with shorter survival in patients with pancreatic cancer, unlike low muscle mass. ${ }^{27}$ This is consistent with our findings. The possible reason is that low SMA induces an inflammatory microenvironment through an imbalance in adipokines and other cytokines, which could impair the immune system and increase the risk of death. ${ }^{28}$

Another influential factor in the development of MSAP or SAP patients was VAT. High deposition of adipose tissue was closely associated with the chronic inflammatory state. Chronic inflammation may impair leptin function producing leptin resistance by interfering in leptin receptor signaling. ${ }^{29}$ The leptin resistance in the hypothalamus impairs the weight control that may lead to obesity. $^{29}$ Due to leptin resistance, the effect of leptin on inhibiting lipid deposition in skeletal muscle is decreased, which may lead to patients with sarcopenia or myosteatosis. Studies have suggested that high VAT was strongly associated with increased infections at the surgical site in patients with pancreatic cancer. ${ }^{30}$ Studies have suggested that the level of serum adiponectin is inversely correlated with abdominal adiposity. ${ }^{31}$ It is known that serum adiponectin exerts anti-inflammatory effects. Adiponectin induces the production of anti-inflammatory mediators in immune cells and inhibits the production of proinflammatory cytokines (TNF- $\alpha$ and IL-6) by macrophages. ${ }^{32}$ Additionally, IL- 6 and TNF- $\alpha$ also inhibit the production of adiponectin by adipocytes and form a vicious cycle. ${ }^{33}$ 
Serum adiponectin also promotes free fatty acid clearance and fatty acid oxidation in mitochondria. ${ }^{34}$ Low levels of adiponectin reduce clearance and fat oxidation of fatty acids, leading to lipotoxicity. It has been reported that adiponectin levels in SAP are significantly lower than those in patients with MAP. ${ }^{31}$ Relevant animal experimental studies have shown that adiponectin therapy can significantly reduce the severity of AP. ${ }^{35}$ For the study of recurrent AP, Table 4 shows that VAT had little change relative to the first measurement and was still at a high level in the recurrent group (156.6 vs $152 \mathrm{~cm}^{2}, \mathrm{p}=0.442$ ). In contrast, VAT was significantly decreased in the nonrecurrent group (144.2 vs $\left.103 \mathrm{~cm}^{2}, \mathrm{p}=0.005\right)$. Recent studies have suggested that abdominal adiposity in AP patients during follow-up was positively correlated with elevated levels of the proinflammatory cytokines IL- 6 and TNF- $\alpha .{ }^{36}$ This was consistent with our research. These data also suggested that a decrease of more than $17 \%$ in VAT within 1 year was a protective factor.

In this study, treatment strategies and health education on diet and exercise after discharge for AP patients are important. The main treatment strategies for AP patients are fasting water, gastrointestinal decompression, acid inhibition, enzyme inhibition and supplemental physiological requirements. For patients with MSAP and SAP, it is important to block SIRS, maintain organ function, analgesia, early enteral nutrition, rational use of antibiotics, adequate treatment of local and systemic complications, and surgical treatment. ${ }^{37}$ In our data, 14 patients with SAP underwent surgical intervention. Finally, the doctor advised the patients to have a proper diet and moderate physical exercise after discharge. It has been reported that intake of vegetables, fish, fiber, and milk may reduce the risk of AP. ${ }^{38-40}$ Results of a prospective cohort study of 0.5 million people showed that there was an inverse association between physical activity and risk of $\mathrm{AP}^{41}{ }^{4}$ It has also been reported that combined aerobic exercise and resistance exercise may reduce the occurrence of sarcopenia obesity and improve muscle function. ${ }^{42}$ It is very important for patients to have a reasonable diet and exercise for the occurrence of the disease and improve the prognosis.

We acknowledge that there are several limitations in our study. First, this study was retrospective in design and lacked the detection of proinflammatory and antiinflammatory cytokines. Second, for recurrent studies, many patients do not undergo re-examination of abdominal CT after recovery, which may lead to certain errors. Third, CT images were measured by one experienced physician. It has been reported that the correlation coefficient between the two measurements may reach 0.954-0.99, so our measurement has little influence on the results. ${ }^{11,14}$ Finally, this study included only a singlecenter population in China. Therefore, further multicenter, large-sample, randomized and prospective studies in different regions would be helpful to verify our findings.

\section{Conclusion}

Visceral adipose tissue and skeletal muscle attenuation were strongly correlated with the severity and prognosis of patients with AP. The impact of VAT and skeletal muscle attenuation on the process and outcome of AP also needs to be further investigated. Last, it is our opinion that patients should receive education on diet and exercise after discharge from the hospital. Proper diet and exercise may increase muscle function and reduce visceral obesity in patients, which is beneficial for improving the quality of life and prognosis of patients.

\section{Abbreviations}

BMI, body mass index; SMT, skeletal muscle tissue; SMA, skeletal muscle attenuation; L3-SMI, lumbar third skeletal muscle index; VAT, visceral adipose tissue; VATA, visceral adipose tissue attenuation; SAT, subcutaneous adipose tissue; SATA, subcutaneous adipose tissue attenuation; HU, Hounsfield Units; OR, odd ratio; CI, confidence interval.

\section{Ethics Approval and Consent to Participate}

The retrospective study procedures were conducted in accordance with the Declaration of Helsinki, and approved by the Ethics Committee of the PLA Rocket Force Characteristic Medical Center, Beijing, China in January 2021 ((KY2021026). The ethics committee did not require participants to sign informed consent. We only obtained body composition data and relevant clinical information without any adverse effects on the participants, and not involved the privacy of patients.

\section{Disclosure}

The authors report no conflicts of interest in this work.

\section{References}

1. Lee PJ, Papachristou GI. New insights into acute pancreatitis. Nat Rev Gastroenterol Hepatol. 2019;16(8):479-496.

2. Masamune A, Kikuta K, Hamada S, et al. Clinical practice of acute pancreatitis in Japan: an analysis of nationwide epidemiological survey in 2016. Pancreatology. 2020;20(4):629-636. doi:10.1016/j.pan.20 20.04.013 
3. Cho JH, Jeong YH, Kim KH, Kim TN. Risk factors of recurrent pancreatitis after first acute pancreatitis attack: a retrospective cohort study. Scand J Gastroenterol. 2020;55(1):90-94. doi:10.1080/ 00365521.2019 .1699598

4. Vege SS, Gardner TB, Chari ST, et al. Low mortality and high morbidity in severe acute pancreatitis without organ failure: a case for revising the Atlanta classification to include "moderately severe acute pancreatitis". Am J Gastroenterol. 2009;104(3):710-715.

5. Sarr MG. 2012 revision of the Atlanta classification of acute pancreatitis. Pol Arch Med Wewn. 2013;123(3):118-124.

6. Cote GA, Yadav D, Abberbock JA, et al. Recurrent acute pancreatitis significantly reduces quality of life even in the absence of overt chronic pancreatitis. Am J Gastroenterol. 2018;113(6):906-912. doi:10.1038/s41395-018-0087-7

7. MacDonald AJ, Greig CA, Baracos V. The advantages and limitations of cross-sectional body composition analysis. Curr Opin Support Palliat Care. 2011;5(4):342-349. doi:10.1097/ SPC.0b013e32834c49eb

8. Leibovitz E, Ben-David N, Shibanov L, Elias S, Shimonov M. Visceral adiposity but not subcutaneous fat associated with improved outcome of patients with acute cholecystitis. $J$ Surg Res. 2018;225:15-20. doi:10.1016/j.jss.2017.11.053

9. Bollen TL, Singh VK, Maurer R, et al. A comparative evaluation of radiologic and clinical scoring systems in the early prediction of severity in acute pancreatitis. Am J Gastroenterol. 2012;107 (4):612-619. doi:10.1038/ajg.2011.438

10. Kuan LL, Dennison AR, Garcea G. Association of visceral adipose tissue on the incidence and severity of acute pancreatitis: a systematic review. Pancreatology. 2020;20(6):1056-1061. doi:10.1016/j. pan.2020.05.027

11. Sternby H, Mahle M, Linder N, et al. Mean muscle attenuation correlates with severe acute pancreatitis unlike visceral adipose tissue and subcutaneous adipose tissue. United European Gastroenterol J. 2019;7(10):1312-1320.

12. van Grinsven J, van Vugt JLA, Gharbharan A, et al. The association of computed tomography-assessed body composition with mortality in patients with necrotizing pancreatitis. $J$ Gastrointest Surg. 2017;21 (6): 1000-1008. doi:10.1007/s11605-016-3352-3

13. Banks PA, Bollen TL, Dervenis C, et al. Classification of acute pancreatitis-2012: revision of the Atlanta classification and definitions by international consensus. Gut. 2013;62(1):102-111. doi:10.1136/gutjnl-2012-302779

14. Woodward AJ, Avery A, Keating SE, Ward LC, Coombes JS, Macdonald GA. Computerised tomography skeletal muscle and adipose surface area values in a healthy Caucasian population. Eur J Clin Nutr. 2020;74(9):1276-1281. doi:10.1038/s41430-020-0628-1

15. Aubrey J, Esfandiari N, Baracos VE, et al. Measurement of skeletal muscle radiation attenuation and basis of its biological variation. Acta Physiol. 2014;210(3):489-497. doi:10.1111/apha.12224

16. Murphy RA, Register TC, Shively CA, et al. Adipose tissue density, a novel biomarker predicting mortality risk in older adults. $J$ Gerontol a Biol Sci Med Sci. 2014;69(1):109-117. doi:10.1093/gerona/glt070

17. Taaffe DR, Henwood TR, Nalls MA, Walker DG, Lang TF, Harris TB. Alterations in muscle attenuation following detraining and retraining in resistance-trained older adults. Gerontology. 2009;55(2):217-223. doi:10.1159/000182084

18. Vella CA, Cushman M, Van Hollebeke RB, Allison MA. Associations of abdominal muscle area and radiodensity with adiponectin and leptin: the Multiethnic Study of Atherosclerosis. Obesity. 2018;26(7):1234-1241. doi:10.1002/oby.22208

19. Hamrick MW. Role of the cytokine-like hormone leptin in muscle-bone crosstalk with aging. $J$ Bone Metab. 2017;24(1):1-8. doi:10.11005/jbm.2017.24.1.1

20. Mechanick JI, Zhao S, Garvey WT. Leptin, an adipokine with central importance in the global obesity problem. Glob Heart. 2018;13 (2):113-127. doi:10.1016/j.gheart.2017.10.003
21. Dyck DJ, Heigenhauser GJ, Bruce CR. The role of adipokines as regulators of skeletal muscle fatty acid metabolism and insulin sensitivity. Acta Physiol. 2006;186(1):5-16. doi:10.1111/j.17481716.2005.01502.x

22. Konturek PC, Jaworek J, Maniatoglou A, et al. Leptin modulates the inflammatory response in acute pancreatitis. Digestion. 2002;65 (3):149-160. doi:10.1159/000064935

23. Jin Z, Xu L, Wang X, Yang D. Risk factors for worsening of acute pancreatitis in patients admitted with mild acute pancreatitis. Med Sci Monit. 2017;23:1026-1032. doi:10.12659/MSM.900383

24. Montano-Loza AJ, Angulo P, Meza-Junco J, et al. Sarcopenic obesity and myosteatosis are associated with higher mortality in patients with cirrhosis. J Cachexia Sarcopenia Muscle. 2016;7(2):126-135. doi:10.1002/jcsm.12039

25. Zhou C, Foster B, Hagge R, et al. Opportunistic body composition evaluation in patients with esophageal adenocarcinoma: association of survival with (18) F-FDGPET/CT muscle metrics. Ann Nucl Med. 2020;34(3):174-181. doi:10.1007/s12149-019-01429-7

26. Veld J, Vossen JA, De Amorim Bernstein K, Halpern EF, Torriani M, Bredella MA. Adipose tissue and muscle attenuation as novel biomarkers predicting mortality in patients with extremity sarcomas. Eur Radiol. 2016;26(12):4649-4655. doi:10.1007/s00330-016-4306-6

27. Rollins KE, Tewari N, Ackner A, et al. The impact of sarcopenia and myosteatosis on outcomes of unresectable pancreatic cancer or distal cholangiocarcinoma. Clin Nutr. 2016;35(5):1103-1109. doi:10.1016/ j.clnu.2015.08.005

28. Hamaguchi Y, Kaido T, Okumura S, et al. Impact of skeletal muscle mass index, intramuscular adipose tissue content, and visceral to subcutaneous adipose tissue area ratio on early mortality of living donor liver transplantation. Transplantation. 2017;101(3):565-574. doi:10.1097/TP.0000000000001587

29. Perez-Perez A, Sanchez-Jimenez F, Vilarino-Garcia T, SanchezMargalet V. Role of leptin in inflammation and vice versa. Int J Mol Sci. 2020;21:16. doi:10.3390/ijms21165887

30. van Dijk DP, Bakens MJ, Coolsen MM, et al. Low skeletal muscle radiation attenuation and visceral adiposity are associated with overall survival and surgical site infections in patients with pancreatic cancer. J Cachexia Sarcopenia Muscle. 2017;8(2):317-326. doi: $10.1002 / \mathrm{jcsm} .12155$

31. Karpavicius A, Dambrauskas Z, Sileikis A, Vitkus D, Strupas K. Value of adipokines in predicting the severity of acute pancreatitis: comprehensive review. World $J$ Gastroenterol. 2012;18 (45):6620-6627. doi:10.3748/wjg.v18.i45.6620

32. Ouchi N, Walsh K. Adiponectin as an anti-inflammatory factor. Clin Chim Acta. 2007;380(1-2):24-30. doi:10.1016/j.cca.2007.01.026

33. Fasshauer M, Kralisch S, Klier M, et al. Adiponectin gene expression and secretion is inhibited by interleukin-6 in 3T3-L1 adipocytes. Biochem Biophys Res Commun. 2003;301(4):1045-1050. doi:10.1016/S0006-291X(03)00090-1

34. Yokota T, Oritani K, Takahashi I, et al. Adiponectin, a new member of the family of soluble defense collagens, negatively regulates the growth of myelomonocytic progenitors and the functions of macrophages. Blood. 2000;96(5):1723-1732. doi:10.1182/blood. V96.5.1723

35. Araki $H$, Nishihara $T$, Matsuda $M$, et al. Adiponectin plays a protective role in caerulein-induced acute pancreatitis in mice fed a high-fat diet. Gut. 2008;57(10):1431-1440. doi:10.1136/ gut.2007.135665

36. Singh RG, Pendharkar SA, Gillies NA, Miranda-Soberanis V, Plank LD, Petrov MS. Associations between circulating levels of adipocytokines and abdominal adiposity in patients after acute pancreatitis. Clin Exp Med. 2017;17(4):477-487. doi:10.1007/ s10238-017-0453-6

37. Li J, Chen J, Tang W. The consensus of integrative diagnosis and treatment of acute pancreatitis-2017. J Evid Based Med. 2019;12 (1):76-88. doi:10.1111/jebm. 12342 
38. Oskarsson V, Sadr-Azodi O, Orsini N, Andren-Sandberg A, Wolk A. Vegetables, fruit and risk of non-gallstone-related acute pancreatitis: a population-based prospective cohort study. Gut. 2013;62 (8):1187-1192. doi:10.1136/gutjnl-2012-302521

39. Oskarsson V, Orsini N, Sadr-Azodi O, Wolk A. Fish consumption and risk of non-gallstone-related acute pancreatitis: a prospective cohort study. Am J Clin Nutr. 2015;101(1):72-78. doi:10.3945/ ajcn.113.076174

40. Koncz B, Darvasi E, Erdosi D, et al. LIFEStyle, Prevention and Risk of Acute PaNcreatitis (LIFESPAN): protocol of a multicentre and multinational observational case-control study. BMJ Open. 2020;10 (1):e029660. doi:10.1136/bmjopen-2019-029660
41. Pang Y, Kartsonaki C, Turnbull I, et al. Metabolic and lifestyle risk factors for acute pancreatitis in Chinese adults: a prospective cohort study of 0.5 million people. PLoS Med. 2018;15(8):e1002618. doi:10.1371/journal.pmed.1002618

42. Dieli-Conwright CM, Courneya KS, Demark-Wahnefried W, et al. Effects of aerobic and resistance exercise on metabolic syndrome, sarcopenic obesity, and circulating biomarkers in overweight or obese survivors of breast cancer: a randomized controlled trial. J Clin Oncol. 2018;36(9):875-883. doi:10.1200/JCO.2017.75.7526

\section{Publish your work in this journal}

The International Journal of General Medicine is an international, peer-reviewed open-access journal that focuses on general and internal medicine, pathogenesis, epidemiology, diagnosis, monitoring and treatment protocols. The journal is characterized by the rapid reporting of reviews, original research and clinical studies across all disease areas. The manuscript management system is completely online and includes a very quick and fair peer-review system, which is all easy to use. Visit http://www.dovepress.com/ testimonials.php to read real quotes from published authors. 